\title{
Deutschlandweites Vergiftungsspektrum im Kindes- und Jugendalter
}

\section{Intoxications in Children and Adolescents in Germany}

\author{
Autoren \\ Stefanie Geith', Martin Ganzert ${ }^{1}$, Sabrina Schmoll1', Daniela Acquarone², Michael Deters ${ }^{3}$, Oliver Sauer ${ }^{4}$, \\ Andreas Stürer ${ }^{4}$, Erol Tutdibi ${ }^{5}$, Rafael Wagner ${ }^{6}$, Florian Eyer ${ }^{1}$
}

\section{Einrichtungen}

1 Abteilung für Klinische Toxikologie \& Giftnotruf München, Klinikum rechts der Isar, Technische Universität München

2 Charité Universitätsmedizin Berlin Campus Benjamin Franklin, Giftnotruf der Charité, Berlin

3 Gemeinsames Giftinformationszentrum der Länder Mecklenburg-Vorpommern, Sachsen, Sachsen-Anhalt und Thüringen c/o HELIOS Klinikum Erfurt, Erfurt

4 Giftinformationszentrum der Länder Rheinland-Pfalz und Hessen - Klinische Toxikologie, Universitätsmedizin Mainz

5 Informations- und Behandlungszentrum für Vergiftungen des Saarlandes, Universitätsklinikum und Medizinische Fakultät der Universität des Saarlandes, Homburg

6 Zentrum 5 Pharmakologie und Toxikologie, Giftinformationszentrum, Georg-August-Universität Gottingen Universitätsmedizin, Göttingen

\section{Schlüsselwörter}

Prävention von Vergiftungen, Erstmaßnahmen im Vergiftungsfall, häufige Noxen, Beratung durch Giftnotrufzentralen

\section{Key words}

prevention of intoxications, initial measures in acute intoxications, frequent toxicants, poison centers consultation

\section{Bibliografie}

DOI https://doi.org/10.1055/a-0594-9480

Online-Publikation: 18.6.2018

Klin Padiatr 2018; 230: 205-214

(C) Georg Thieme Verlag KG Stuttgart · New York ISSN 0300-8630

\author{
Korrespondenzadresse \\ Dr. Stefanie Geith \\ Abteilung für Klinische Toxikologie \\ Giftnotruf München \\ Klinikum rechts der Isar \\ Technische Universität München \\ Ismaninger Straße 22 \\ 81675 München \\ Tel.: +49/89/4140 2241, Fax: +49/89/41404789 \\ stefanie.geith@gmx.de
}

\section{ZUSAMMENFASSUNG}

Hintergrund Vergiftungen verursachen bei Kindern den Großteil der Notfalleinsätze in Deutschland, die durch präventive Maßnahmen verhindert oder gemildert werden könnten. Daher ist für den Pädiater das Wissen um häufige Intoxikationen essentiell. Die vorliegende Arbeit zeigt allgemeine und epidemiologische Daten zu Vergiftungen sowie einen Überblick über die häufigsten einzelnen Noxen und -kategorien im Kindes- und Jugendalter.

Methoden Retrospektiv wurden Vergiftungsfälle bei Kindern und Jugendlichen aus 6 deutschen Giftnotrufzentralen (20122016 und 2002-2016) ausgewertet. Kategorielle Daten sind als Mittelwerte \pm Standardabweichung, häufigste Noxen nach Punkten angegeben.

Ergebnisse Die Anruferzahl insbesondere der Laien nahm ab 2002 deutlich zu. Zwei Drittel der Fälle traten bei Klein- und Vorschulkindern auf, häufiger bei Jungen ( $50 \%$ ) als bei Mädchen (44\%), bei Jugendlichen überwiegen weibliche Patienten (>60\%). Im Alter < 14 Jahre sind Intoxikationen auf Unfälle in Haushalt, Kindertagespflege oder Schulen zurückzuführen (>95\%), bei Jugendlichen treten suizidale Intoxikationen und Abusus (13\%) in den Vordergrund. 90 \% der Fälle verlaufen asymptomatisch oder leicht, wobei der Anteil der klinisch symptomatischen Fälle mit dem Alter zunimmt (Jugendliche 13\% vs. Säuglinge $1 \%$ ). Vergiftungen mit Medikamenten stellen bei Jugendlichen die häufigste Gruppe dar, bei Kindern tensidhaltige Reinigungsmittel und Kosmetika, Sanitärreinigungsmittel, Tabak, Knicklicht und Entkalker in Lösung.

Diskussion und Schlussfolgerung Stetig steigende Anruferzahlen von Fachpersonal und Laien veranschaulichen die Bedeutung der Giftnotrufzentralen. Obwohl Vergiftungen bei Kindern und Jugendlichen meist asymptomatisch oder mit leichten Symptomen verlaufen, darf die Relevanz präventiver Maßnahmen v. a. bei Kindern $<7$ Jahren nicht unterschätzt werden.

\section{ABSTRACT}

Background In Germany, intoxications cause the bulk of emergencies in children, to be prevented or attenuated by preventive measures. Therefore, knowledge about intoxications is essential for pediatricians. The present work provides gene- 
ral and epidemiologic data about intoxications and most frequent categories and single toxicants.

Methods Data of intoxications in children and adolescents from 6 German poison centers (2012-2016 and 2002-2016) were retrospectively analyzed. Categorical data are given as mean \pm standard deviation, most frequent toxicants as a score. Results Calls, especially from non-professionals, increased since 2002. Two third of intoxications occurred in small and pre-school children, more frequently in boys $(50 \%)$ than girls (44\%), in adolescents girls predominated $(>60 \%) .<14$ years intoxications occur mainly at home, day care or school (>95\%), in adolescents suicide attempts and abuse come to the fore
(13\%). $90 \%$ of the cases are asymptomatic or mild, with increasing symptoms at higher ages (adolescents $13 \%$ vs. small children $1 \%$ ). Intoxications with drugs are predominantly in adolescents, surfactant containing cleaning agents and cosmetics, sanitary cleaner, tobacco, glow lights and solute descaler in children.

Discussion and Conclusions Increasing incoming calls from professionals and non-professionals point out the importance of the poison centers. Although intoxications in children and adolescents mainly proceed without or mild symptoms, the relevance of preventive measures especially for children $<7$ should not be underestimated.

\section{Hintergrund}

Akute Vergiftungsfälle machen ca. 5-10\% der Notfalleinsätze in Deutschland aus $[5,10]$, wobei in ungefähr der Hälfte der Fälle Kinder und Jugendliche betroffen sind $[6,8]$. Nicht selten ist die erste Anlaufstelle besorgter Eltern der behandelnde Kinderarzt, in dessen Verantwortlichkeit die Entscheidung über das weitere Vorgehen liegt. Umso wichtiger ist für den Pädiater das Wissen um die in der Altersgruppe < 18 Jahre häufigsten Noxen, deren Risikoeinschätzung und Initialtherapie. Darüberhinaus kommt dem Pädiater auch eine wichtige Rolle bei der Aufklärung der Eltern seiner Patienten über primär- und sekundärpräventive Vorkehrungen zu, die gerade in der Gruppe der (Vor-)Schulkinder einen Hauptansatzpunkt darstellen, um die Anzahl der Vergiftungen zu verringern oder deren Schwere in ihrem Verlauf zu mildern. \ Abb. 1 gibt einen Überblick über mögliche Präventionsmaßnahmen, Verfah- ren der primären und sekundären Giftelimination, sowie Erstmaßnahmen im Vergiftungsfall. Verfahren der primären Giftentfernung dienen der Verringerung bzw. Verhinderung der Giftresorption und orientieren sich nach der Aufnahmepforte des Giftes. Im Falle einer oralen Giftaufnahme ist die nebenwirkungsarme Gabe von 0,5-1 g/ kg Körpergewicht Aktivkohle das Mittel der ersten Wahl - sofern die aufgenommenen Giftstoffe an Aktivkohle binden. Alkohole, Säuren, Laugen, Metalle und anorganische Ionen werden nicht ausreichend von Aktivkohle absorbiert und stellen damit die seltene Ausnahme dar, bei der eine Kohlegabe nicht sinnvoll ist. Demgegenüber bleibt das Auslösen von Erbrechen wie auch die Magenspülung angesichts möglicher schwerer Nebenwirkungen (z. B. Aspiration, Pneumonie), wenigen Ausnahmefällen unter strenger Indikationsstellung nach Rücksprache mit einer Giftnotrufzentrale (GIZ) vorbehalten [1, 3, 13]. In diesem Zusammenhang sei auch auf

\begin{tabular}{|c|c|c|c|c|c|c|c|c|}
\hline PRIMÄRPRÄVENTION & \multicolumn{4}{|c|}{ SEKUNDÄRPRÄVENTION } & \multicolumn{4}{|c|}{ TERTIÄRPRÄVENTION } \\
\hline $\begin{array}{l}\text { Maßnahmen zur Verhinderung des } \\
\text { Auftretens einer Vergiftung }\end{array}$ & \multicolumn{4}{|c|}{$\begin{array}{l}\text { Maßnahmen zur Verhinderung des Voranschreitens bzw. zur Minderung der Schwere einer } \\
\text { bereits erfolgten Vergiftung/nach stattgehabter Exposition }\end{array}$} & \multicolumn{4}{|c|}{$\begin{array}{l}\text { Maßnahmen, die bei Intoxikierten durchgeführt werden können, um den Tod oder eine } \\
\text { permanente Behinderung zu verhindern, bzw. ihr vorzubeugen }\end{array}$} \\
\hline VERHALTENSPRÄVENTION & \multicolumn{4}{|c|}{ PRIMÄRE GIFTELIMINATION } & \multicolumn{4}{|c|}{ SERUNDÄRE GIFTELIMINATION } \\
\hline \multirow{3}{*}{$\begin{array}{l}\text { Abgabe von Medikamenten in kleineren } \\
\text { Packungsgrößen } \\
\text { Ersatz toxischer durch weniger toxische } \\
\text { Substanzen }\end{array}$} & \multicolumn{4}{|c|}{ Verhinderung/Minderung/Verzögerung der Giftresorption } & \multicolumn{4}{|c|}{ Entfernung des Fremdstoffes aus dem Kreislaufsystem nach dessen Resorption } \\
\hline & $\begin{array}{l}\text { ORALE } \\
\text { AUFNAHME }\end{array}$ & Adsorbens & Aktivkohle & $\begin{array}{l}\text { o,5-1 } \mathrm{g} / \mathrm{kgKG} \text {; } \\
\text { max. } 50 \mathrm{~g} \text { Einmaldosis }\end{array}$ & $\begin{array}{l}\text { REPETITTVE } \\
\text { KOHLEGABE }\end{array}$ & Aktivkohle & $50 \mathrm{~g}$ alle $4 \mathrm{~h}$ & $\begin{array}{l}\text { Carbamazepin, } \\
\text { Dapson, Chinin, } \\
\text { Theophyllin, } \\
\text { Phenobarbital }\end{array}$ \\
\hline & & Magenspülung & $\begin{array}{l}\text { Kochsalzlösung } \\
\text { (über }\end{array}$ & $\begin{array}{l}\text { rep. 5-1O ml/ } / \mathrm{kgKG} \mathrm{H}_{2} \mathrm{O} \\
\text { gesamt } 15-2 \mathrm{Ol} \mathrm{H}_{2} \mathrm{O}\end{array}$ & $\begin{array}{l}\text { FORCIERTE } \\
\text { DIURESE } \\
\text { evtl. plus }\end{array}$ & $\begin{array}{l}\text { Kristalloide } \\
\text { Furosemid }\end{array}$ & $\begin{array}{l}300-500 \mathrm{ml} / \mathrm{h} \\
20-40 \mathrm{mg} \text { i.v. }\end{array}$ & $\begin{array}{l}\text { Lithium } \\
\text { Salizylate }\end{array}$ \\
\hline \multirow[t]{4}{*}{$\begin{array}{l}\text { - Bevorratung von Aktivkohle, Dimeticon } \\
\text { - Verhaltensänderung im Umgang mit } \\
\text { potentiellen Gefahrenstoffen im } \\
\text { Haushalt }\end{array}$} & $\begin{array}{l}\text { Ausnahme- } \\
\text { fällen } \\
\text { indiziert }\end{array}$ & $\begin{array}{l}\text { Osmotisches } \\
\text { Laxans }\end{array}$ & Na-Sulfat & $15-30 \mathrm{~g}$ auf $10 \mathrm{OO}-15 \mathrm{O} \mathrm{ml} \mathrm{H}_{2} \mathrm{O}$ & \multirow{4}{*}{$\begin{array}{l}\text { HARN- } \\
\text { ALKALISIERUNG } \\
\text { EXTRAKORPO- } \\
\text { RALE VERFAHREN }\end{array}$} & NaBic 8,4\% & \multirow[t]{2}{*}{$1 \mathrm{ml} / \mathrm{kg} / \mathrm{h}$} & \multirow{4}{*}{$\begin{array}{l}\text { Barbiturate } \\
\text { Salizylate } \\
\text { - Plasmapherese }\end{array}$} \\
\hline & INHALATIV & \multicolumn{3}{|c|}{ Entfernung aus dem Gefahrenbereich, $\mathrm{O}_{2}-/$ Frischluft-zufuhr } & & & & \\
\hline & EPIDERMAL & \multicolumn{3}{|c|}{ Abwaschen mit Seife, Entfernen kontaminierter Kleidung } & & - Hämoperfusion & $\begin{array}{l}\text { - Eiweißdialyse } \\
\text {. }\end{array}$ & \\
\hline & OKULÄR & \multicolumn{3}{|c|}{ 1o Minuten Augenspülung unter fließendem Wasser } & & & & \\
\hline \multicolumn{9}{|c|}{ SICHERUNG DER VITALFUNKTIONEN } \\
\hline Atemwege freimachen & & \multicolumn{7}{|c|}{ Substanzreste, Erbrochenes, trockene Schleimhäute, Speichelfluss, Verätzungen } \\
\hline Beatmen & & \multicolumn{7}{|c|}{ Atemgeruch, Atemfrequenz/-tiefe, Auskultation/Perkussion, $\mathrm{spO}_{2}$, Atemgeräusche, Thoraxbewegungen } \\
\hline Kardiopulmonale Reani & mation & \multicolumn{7}{|c|}{ HF, RR, kapilläre Rezirkulation, Körpertemperatur, Farbe/Temperatur der Extremitäten } \\
\hline Bewusstsein, Neurostat & & \multicolumn{7}{|c|}{ Vigilanz, GCS, Agitiertheit, Koma, Halluzinationen, Pupillengröße/-reagibilität, Reflexe, Muskeltonus, Motorik, Seh-/Hörstörungen, Fokalneurologie } \\
\hline Umgebende Faktoren, $\mathrm{E}$ & xploration & \multicolumn{7}{|c|}{ Einstichstellen, Hautveränderungen, Auffindesituation, Asservate } \\
\hline
\end{tabular}

- Abb. 1 Präventionsmaßnahmen zielen darauf ab, Vergiftungen zu vermeiden bzw. nach Intoxikation eine Ausbreitung im Körper zu vermeiden. Im akuten Vergiftungsfall hat zunächst die Sicherung der Vitalfunktionen nach dem $A B C(D E)$-Schema oberste Priorität. Danach folgen primäre und sekundäre Gifteliminationsmaßnahmen. 
die Homepage der Gesellschaft für Klinische Toxikologie www. klinitox.de verwiesen, auf der die Kontaktdaten sämtlicher GIZen Deutschlands, Österreichs und der Schweiz aufgeführt sind. Nach der zunächst symptomatischen Sicherung der Vitalfunktionen gemäß dem in - Abb. 1 dargestellten $A B C(D E)$-Schema nimmt in der Präklinik die Asservierung potentiell ingestierter Noxen (z. B. Tablettenblister, Gefäße von Putz-Reinigungsmitteln, Pilzreste, usw.) sowie die Antidottherapie eine zentrale Stellung ein. Diese kann - abhängig von der eingenommenen Substanz und dem klinischen Bild - nach Rücksprache mit einer GIZ (hier: Angabe von Alter, Körpergewicht des Patienten/Kindes, sowie Art, Menge und Zeitpunkt der Aufnahme der toxischen Substanz) sowohl innerklinisch von ärztlichem Personal, im Falle einfach zu handhabender und nebenwirkungsarmer Antidota, wie Aktivkohle und Entschäumer, aber auch präklinisch von Laien oder (geschulten) Ersthelfern verabreicht werden.

\section{Rationale und Zielsetzung der vorliegenden Arbeit}

Derzeit existieren in Deutschland GIZen in Berlin, Bonn, Erfurt, Freiburg, Göttingen, Homburg, Mainz und München. Seit 1995 sind alle GIZen aufgrund des Beschlusses des Europarates (Resolution 90/C329/03, rev. 24.11.95) angehalten, als Datengrundlage für länderübergreifende Verbraucherschutzinitiativen jährliche Berichte über ihre Tätigkeit zu erstellen [2].

Ziel der aktuellen Untersuchung ist es, über einen Zeitraum von 5 Jahren (2012-2016) retrospektiv Daten zu Vergiftungsfällen bei Kindern und Jugendlichen aus verschiedenen GIZen Deutschlands überregional auszuwerten. Zusätzlich zu allgemeinen Basisdaten der Vergiftung sollen dabei altersgruppenspezifisch die häufigsten Noxen identifiziert und exemplarisch Therapieempfehlungen für besonders häufige Vergiftungen gegeben werden. Schließlich soll eine isolierte Auswertung der Münchner Datenbank über einen längeren Zeitraum von 2002 bis 2016 im Hinblick auf die jährlichen Anruferzahlen sowie das Vorhandensein klinischer Symptome ungeachtet deren Schweregrades einen längerfristigen zeitlichen Verlauf dieser Parameter aufzeigen.

\section{Methoden}

Für die vorliegende retrospektive Datenerhebung wurden Daten aus 6 GIZen in München, Berlin, Erfurt, Göttingen, Homburg (nur die Jahre 2013-2016) und Mainz ausgewertet. Mittels einer Datenbankabfrage wurden jeweils für definierte Altersgruppen (Säuglinge $\leq 12$ Monate, Klein- und Vorschulkinder ab 13 Monate bis $\leq 6$ Jahre, Schulkinder ab 7 Jahre bis $\leq 13$ Jahre, Jugendliche ab 14 Jahre bis $\leq 17$ Jahre) Geschlecht, Akuität der Vergiftung, Ätiologie der Vergiftung, Ingestionsweg und Schweregrad, sowie eine Auflistung aller beteiligten Hauptnoxen mit ihrer Häufigkeit erhoben. In die Abfrage wurden alle Fälle eingeschlossen, bei denen das Alter der betroffenen Person < 18 Jahren war. Zusätzlich hierzu wurde für die GIZ München die Anzahl der Anrufe zu den Altersgruppen < 18 Jahre im Zeitraum von 2002-2016 mit Angabe der Anrufergruppe (Laien, Kliniken, niedergelassene Ärzte, Rettungsleitstellen, Apotheken oder Sonstige (z. B. Altenheime, Behinderteneinrichtungen, Polizei, Medien, Militär usw.)) und mutmaßlichem Schweregrad der Symptomatik abgefragt.
Die Daten wurden in Excel erfasst und ausgewertet. Kontinuierliche, normal-verteilte Daten wurden als Mittelwerte mit Standardabweichung angegeben. Die kategorischen Daten zu Anruferzahl, Geschlecht, Akuität, Ätiologie, Ingestionsweg und Schweregrad wurden als Anzahl oder Prozentsatz angegeben. Für die Gesamtauswertung wurden die häufigsten an Intoxikationen im Kindesund Jugendalter beteiligten Einzelsubstanzen pro GIZ unter Berücksichtigung der zwischen den einzelnen GIZen stark variierenden Anruferzahlen in eine absteigende Reihenfolge gebracht. Dabei erhielten die jeweils 10 häufigsten Substanzen Punktwerte von 10 bis 1. Die Punktwerte dieser jeweils 10 häufigsten Noxen wurden summiert und der Durchschnittswert gebildet.

\section{Ergebnisse}

Die Datenbankabfragen ergaben insgesamt ca. 426000 Anrufe zu Vergiftungsfällen bei Kindern und Jugendlichen in den Jahren 2012 bis 2016 aus den genannten sechs GIZen in München, Berlin, Erfurt, Göttingen, Mainz und Homburg (hier nur 2013-2016) (> Tab. 1). In der GIZ München gingen in den Jahren 2002 bis 2016 ca. 226500 Anrufe (Alter < 18 Jahre) ein, wobei über die Jahre eine deutliche Zunahme der Anruferzahlen (Alter<18 Jahren) von $12899 \mathrm{im} \mathrm{Jahr}$ 2002 auf 18592 in 2016 zu verzeichnen ist, die im Wesentlichen auf die stetig wachsende Anzahl der Anrufe durch Laien, deren Anteil an der Anruferzahl (Alter<18 Jahre) von 70\% im Jahr 2002 auf $86 \%$ in 2016 anstieg, zurückzuführen ist ( $\mathbf{A b b}$. 2a).

Ca. zwei Drittel der Anrufe betreffen die Altersgruppe der Kleinund Vorschulkinder (zwischen 13 Monaten und einschließlich 6 Jahren). Unter den Altersgruppen bis $<14$ Jahren sind mehrheitlich Jungen betroffen (Säuglinge 49,0\%, Klein-/Vorschulkinder 49,7\%, Schulkinder $49,4 \%$ männlich vs. $43,6 \%, 44,0 \%$ und $44,1 \%$ weiblich), bei den Jugendlichen $\geq 14$ Jahren dominieren die Mädchen (31,0\% männlich vs. $62,5 \%$ weiblich) ( Tab. 1 ). Während sich die Vergiftungsfälle in den Altersgruppen bis < 14 Jahren größtenteils akzidentiell (Säuglinge 95,6\%, Kleinkinder 96,8\%) bzw. ab dem 2 . Lebensjahr in geringerem Maße auch im „gewerblichen“ Umfeld von KITA, Kindergarten oder Schule ereignen (Klein-/ Vorschulkinder $0,5 \%$, Schulkinder 2,5\%, Jugendliche 2,0\%), treten bei den Jugendlichen zunehmend Intoxikationen in (para-) suizidaler Absicht $(46,2 \%)$ oder im Rahmen eines Abusus (12,8\%) in den Vordergrund. latrogen bedingte Intoxikationen oder Vergiftungserscheinungen auf dem Boden von Nebenwirkungen verteilen sich nahezu gleichmäßig auf alle Altersgruppen (Säuglinge 0,26\%, Klein-/Vorschulkinder $0,16 \%$, Schulkinder $1,0 \%$, Jugendliche $1,2 \%$ ), wohingegen der Anteil der (mutmaßlichen) Fremdbeibringungen in den Altersgruppen der $\geq 7$ bis < 18-jährigen dominiert. Was den vermuteten Schweregrad der Vergiftung betrifft, so verläuft der überwiegende Teil der Vergiftungsfälle asymptomatisch oder mit nur leichten Symptomen (Säuglinge 99\%, Klein-/Vorschulkinder 98,5\%, Schulkinder 95,5\%, Jugendliche 87,1\%). Lediglich sehr wenige Fälle zeigen einen schweren Verlauf oder gar letalen Ausgang. Allerdings nimmt mit steigendem Alter der Anteil an klinisch symptomatischen Vergiftungen zu ( $\triangleright$ Tab. 1), was auch die Münchner Daten während des längeren Beobachtungszeitraumes von 2002 bis 2016 bestätigen (mit mittlerer und schwerer Symptomatik: Säuglinge $0,1 \%$, Klein-/Vorschulkinder $0,2 \%$, Schulkinder $0,9 \%$, Jugendliche 4,7\%). Hierzu bleibt jedoch anzumerken, dass die Erfassung des 
- Tab. 1 Tabellarische Übersicht der gesammelten und ausgewerteten Daten aus den Giftnotrufzentralen München, Berlin, Homburg, Erfurt, Göttingen und Mainz.

\begin{tabular}{|c|c|c|c|c|c|c|c|c|c|}
\hline \multicolumn{2}{|l|}{ Kategorie } & \multicolumn{2}{|c|}{ Säuglinge } & \multicolumn{2}{|c|}{ Kleinkinder } & \multicolumn{2}{|c|}{ Schulkinder } & \multicolumn{2}{|c|}{ Jugendliche } \\
\hline & & Mittelwert & SD & Mittelwert & SD & Mittelwert & SD & Mittelwert & SD \\
\hline \multirow{2}{*}{$\begin{array}{l}\text { Durchschnittliche } \\
\text { Anruferzahlen pro } \\
\text { Jahr }\end{array}$} & München & 3469 & $-{ }^{*}$ & 11372 & $-^{*}$ & 1188 & $-^{*}$ & 906 & $-^{*}$ \\
\hline & Gesamt & 14212 & 1582 & 57965 & 6696 & 6828 & 788 & 6039 & 817 \\
\hline \multirow[t]{3}{*}{ Geschlecht } & Männlich & $49,0 \%$ & $3,5 \%$ & $49,7 \%$ & $3,0 \%$ & $49,4 \%$ & $3,4 \%$ & $31,0 \%$ & $5,7 \%$ \\
\hline & Weiblich & $43,6 \%$ & $3,6 \%$ & $44,0 \%$ & $2,4 \%$ & $44,1 \%$ & $3,8 \%$ & $62,5 \%$ & $9,7 \%$ \\
\hline & Unbekannt & $7,3 \%$ & $6,8 \%$ & $6,3 \%$ & $5,2 \%$ & $6,6 \%$ & $6,0 \%$ & $6,5 \%$ & $10,2 \%$ \\
\hline \multirow[t]{9}{*}{ Ätiologie } & Akzidentell & $95,6 \%$ & $5,5 \%$ & $96,8 \%$ & $5,3 \%$ & $84,5 \%$ & $6,7 \%$ & $28,5 \%$ & $11,7 \%$ \\
\hline & Gewerblich & $0,05 \%$ & $0,06 \%$ & $0,5 \%$ & $0,9 \%$ & $2,5 \%$ & $3,6 \%$ & $2,0 \%$ & $1,4 \%$ \\
\hline & Abusus & $0,5 \%$ ** & $1,11 \%$ & $0,4 \%$ ** & $1,0 \%$ & $1,6 \%$ & $1,0 \%$ & $12,8 \%$ & $3,2 \%$ \\
\hline & (Para)-Suizidal & $0,004 \%{ }^{* *}$ & $0,009 \%$ & $0,05 \% * *$ & $0,08 \%$ & $5,5 \%$ & $3,2 \%$ & $46,2 \%$ & $15,0 \%$ \\
\hline & latrogen & $0,5 \%$ & $0,3 \%$ & $0,15 \%$ & $0,05 \%$ & $0,8 \%$ & $0,3 \%$ & $0,7 \%$ & $0,4 \%$ \\
\hline & Nebenwirkung & $0,26 \%$ & $0,25 \%$ & $0,16 \%$ & $0,1 \%$ & $1,0 \%$ & $0,4 \%$ & $1,2 \%$ & $0,3 \%$ \\
\hline & Fremdbeibringung & $0,14 \%$ & $0,1 \%$ & $0,04 \%$ & $0,02 \%$ & $0,9 \%$ & $0,5 \%$ & $2,0 \%$ & $2,1 \%$ \\
\hline & Sonstige & $1,0 \%$ & $2,4 \%$ & $1,0 \%$ & $2,4 \%$ & $1,3 \%$ & $3,2 \%$ & $1,1 \%$ & $2,8 \%$ \\
\hline & Unbekannt & $2,0 \%$ & $3,2 \%$ & $0,9 \%$ & $2,1 \%$ & $2,0 \%$ & $3,1 \%$ & $5,6 \%$ & $8,8 \%$ \\
\hline \multirow[t]{6}{*}{ Schweregrad } & Asymptomatisch & $83,5 \%$ & $13,5 \%$ & $77,0 \%$ & $9,2 \%$ & $57,7 \%$ & $11,2 \%$ & $32,7 \%$ & $9,5 \%$ \\
\hline & Leicht & $15,5 \%$ & $8,7 \%$ & $21,5 \%$ & $7,5 \%$ & $37,8 \%$ & $9,4 \%$ & $54,4 \%$ & $11,3 \%$ \\
\hline & Mittel & $0,8 \%$ & $2,8 \%$ & $1,3 \%$ & $2,9 \%$ & $3,8 \%$ & $6,4 \%$ & $11,0 \%$ & $6,3 \%$ \\
\hline & Schwer & $0,15 \%$ & $3,9 \%$ & $0,12 \%$ & $1,8 \%$ & $0,49 \%$ & $3,0 \%$ & $1,8 \%$ & $11,0 \%$ \\
\hline & Fatal/letal & $0,009 \%$ & $0,006 \%$ & $0,002 \%$ & $0,002 \%$ & $0,004 \%$ & $0,004 \%$ & $0,035 \%$ & $0,5 \%$ \\
\hline & Unbekannt & $0,12 \%$ & $4,0 \%$ & $0,13 \%$ & $4,0 \%$ & $0,2 \%$ & $4,7 \%$ & $0,11 \%$ & $6,1 \%$ \\
\hline
\end{tabular}

* Bei der Sammlung der Daten wurden die durchschnittlichen Anruferzahlen angegeben, daher kann keine Standardabweichung zwischen den Einzelwerten angegeben werden; ${ }^{* *}$ Die Angaben der Vergiftungsfälle bei Säuglingen und Kleinkindern aufgrund eines Abusus oder in (para-)suizidaler Absicht sind vermutlich auf eine fehlerhafte Übertragung der im Giftnotrufprotokoll abgefragten Daten in das Datenerfassungsprogramm zurückzuführen. SD=Standardabweichung

mutmaßlichen Schweregrades einer Vergiftung lediglich auf dem telefonischen Erstkontakt im Rahmen der akuten Vergiftung, d.h. ohne Berücksichtigung des weiteren klinischen Verlaufs bzw. Ausgangs einer Intoxikation beruht und somit ein nicht unerhebliches Maß an Unwägbarkeit bzgl. des tatsächlichen Schweregrades der Vergiftung aufweist.

Betrachtet man die Häufigkeitsverteilung der großen Noxenkategorien auf die einzelnen Altersgruppen, so bilden Medikamente, Pflanzen und Pilze, Reinigungsmittel, Chemikalien und Kosmetika die Hauptgruppen der Vergiftungen bei Säuglingen, Klein-/Vorschulkindern und Schulkindern, wobei die Medikamente mit zunehmendem Alter einen größeren Stellenwert einnehmen und bei den Jugendlichen neben den Rausch- und Suchtmitteln (10\% der Anfragen), mit 67\% die mit Abstand häufigste angefragte Gruppe darstellen. ( $\triangleright$ Abb. $\mathbf{2 b}-\mathbf{e})$

Bei den einzelnen Noxen machen tensidhaltige Reinigungsmittel die häufigsten Vergiftungen in den Altersgruppen der Säuglinge und Kleinkinder aus und stehen auch bei den Schulkindern und Jugendlichen an vorderer Stelle. Tensidhaltige Kosmetika und Sanitärreinigungsmittel, Tabak, Knicklicht und Entkalker in Lösung spielen ebenfalls in den Altersgruppen bis < 14 Jahren eine große Rolle. In der Gruppe der Medikamente überwiegen bei Säuglingen und Kleinkindern Vergiftungen mit Nasentropfen und Schilddrüsenhormonen, während hingegen ab dem Schulalter zunächst insbesondere Schmerzmittel, ab dem 14. Lebensjahr neben den An- algetika auch Psychopharmaka und Sedativa in den Vordergrund rücken. ( $\triangleright$ Abb. $\mathbf{2 f}-\mathbf{g}$ )

- Tab. 2 enthält eine exemplarische Auflistung dieser häufigen Substanzen und gibt Hinweise zur toxischen Dosis, zu möglichen Symptomen und etwaigen Therapiemaßnahmen entsprechend den Standards der GIZ München.

\section{Diskussion}

Die Münchner Verlaufsdaten aus den Jahren 2002 bis 2016 weisen in den letzten Jahren durchwegs einen über $80 \%$-igen Anteil von Laien an einer insgesamt stetig steigenden Anruferzahl zu Vergiftungen im Säuglings-, Kindes- und Jugendalter von $12899 \mathrm{im}$ Jahr 2002 auf 18592 in 2016 auf. Diese Zahlen veranschaulichen eindrucksvoll die gerade in diesen Altersgruppen wachsende Bedeutung der GIZen für die toxikologische Beratung von medizinischem Fachpersonal, in zunehmendem Maße jedoch auch von Laien, respektive besorgten Eltern. Dieser Trend einer kontinuierlich ansteigenden Gesamtanruferzahl (Alter < 18 Jahre) mit einem immer größer werdenden Anteil an Laienanrufern wird sowohl deutschlandweit, als auch im deutschsprachigen Ausland durch die Jahresberichte der Stiftung Tox Info Suisse [12], sowie Daten aus Österreich (GIZ Wien, persönliche Mitteilung) bestätigt. Über die Ursachen dieses Anstieges kann nur spekuliert werden, eine mögliche Erklärung hierfür mag ein in Zeiten der allgegenwärtigen 
a

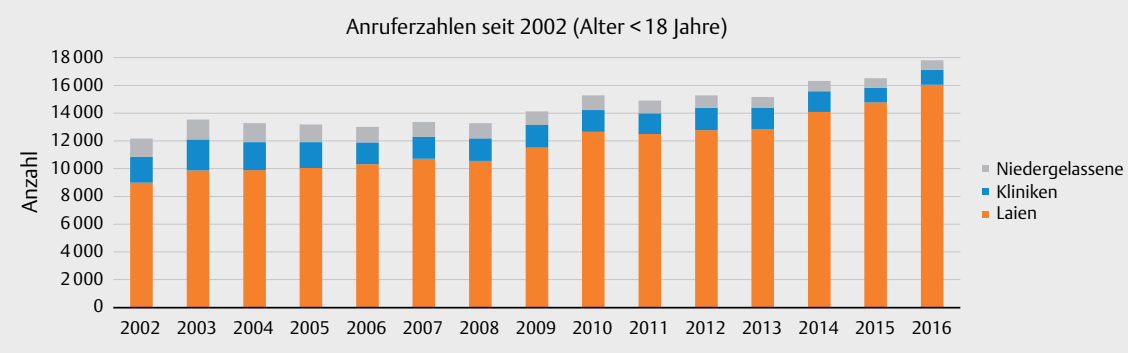

b
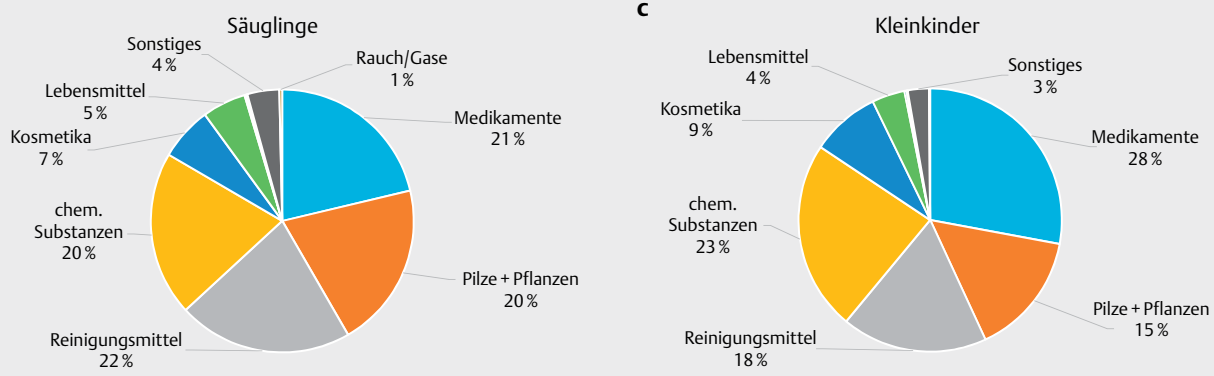

d

Rausch/Suchtmittel 0,7\% Schulkinder

Tierbiss/Stich 2\%— Sonstiges Rauch/Gase

Lebensmittel

$6 \%$ Rauch/Gase

18

e

Rausch/Suchtmittel $5 \%$ Jugendliche Sonstiges

Tierbiss/Stich 0,7\%

Lebensmittel

Kosmetika

$4 \%$

chem.

Substanzen

$\rightarrow$

$6 \%$

$1 \%$

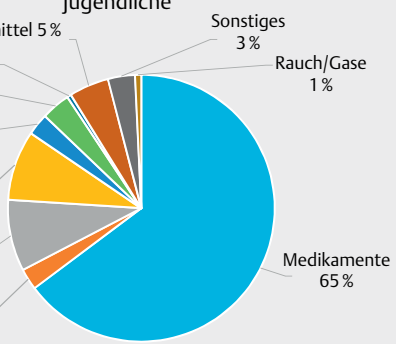

Reinigungsmittel

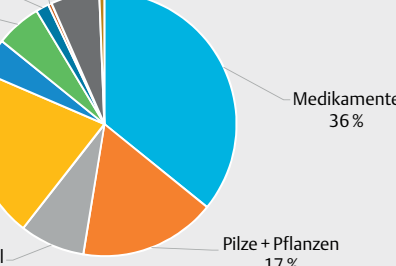

$3 \%$

Kosmetik
$3 \%$

chem. Substanzen

$8 \%$

Reinigungsmittel

$9 \%$

Pilze + Pflanzen

$3 \%$

f

Säuglinge

$\begin{array}{lllllllllll}0 & 1 & 2 & 3 & 4 & 5 & 6 & 7 & 8 & 9 & 10\end{array}$

Tensidhaltige Reinigungsmittel

$$
\text { Tabak }
$$
Entkalker

g

Kleinkinder

$\begin{array}{lllllllllll}0 & 1 & 2 & 3 & 4 & 5 & 6 & 7 & 8 & 9 & 10\end{array}$

Tensidhaltige Sanitärreinigungsmittel Oxalathaltige Pflanzen

Nasentropfen

Schilddrüsenhormone

Körige Kosmetika

Pflanzen gesamt

Ibuprofen

Fremdkörper
Fikus

Grillanzünder

Orchidee

Paracetamol

Tensidhaltige Reinigungsmitte

Tabak
Tensidhaltige Sanitärreinigungsmittel
Ibuprofen

Knicklich

glykosidhaltige Pflanzen

Desinfektionsmittel

Beere rot

Grillanzünder
Fremdkörper

Seifenblasenflüssigkeit

Schilddrüsenhormone

legemittel

Globuli

Nasentropfen
Nagellackentfemer

h

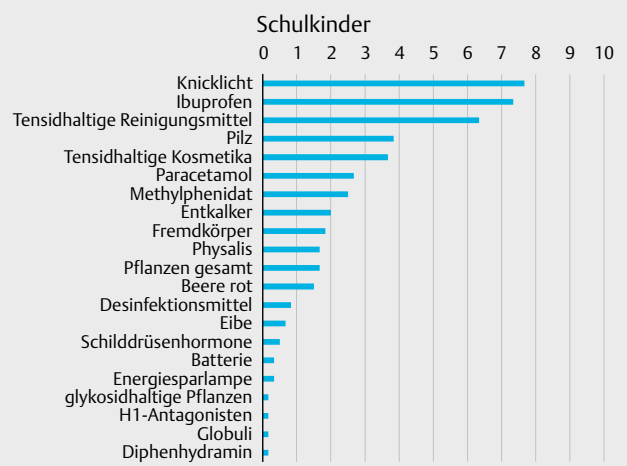

i

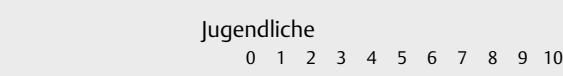

Ibuprofen Paracetamol
Methylphenidat

Tensidhaltige Reinig Ethanol

Fluoxetin

Quetiapin

Entkalker

Knicklich

Mirtazapin
Biss/Stich

Citalopram

H1-Antagonisten

Diphenhydramin

tionsmittel

Schilddrüsenhormone

Nagellackentferner
Pilz

Abb. 2 Dargestellt sind a die Entwicklung der Anruferzahlen für < 18 ährige von Laien (orange), Kliniken (blau) und Niedergelassenen (grau) seit 2002 (Münchner Daten, 2002-2016), b-e die Häufigkeiten der Vergiftungsfälle nach Kategorien (Kuchendiagramme) je Altersgruppe bei Kindern und Jugendlichen und $\mathbf{f}-\mathbf{i}$ die häufigsten Einzelnoxen (Balkendiagramme) je Altersgruppe bei Kindern und Jugendlichen (Daten aus 6 teilnehmenden GIZ, 2012-2016). 


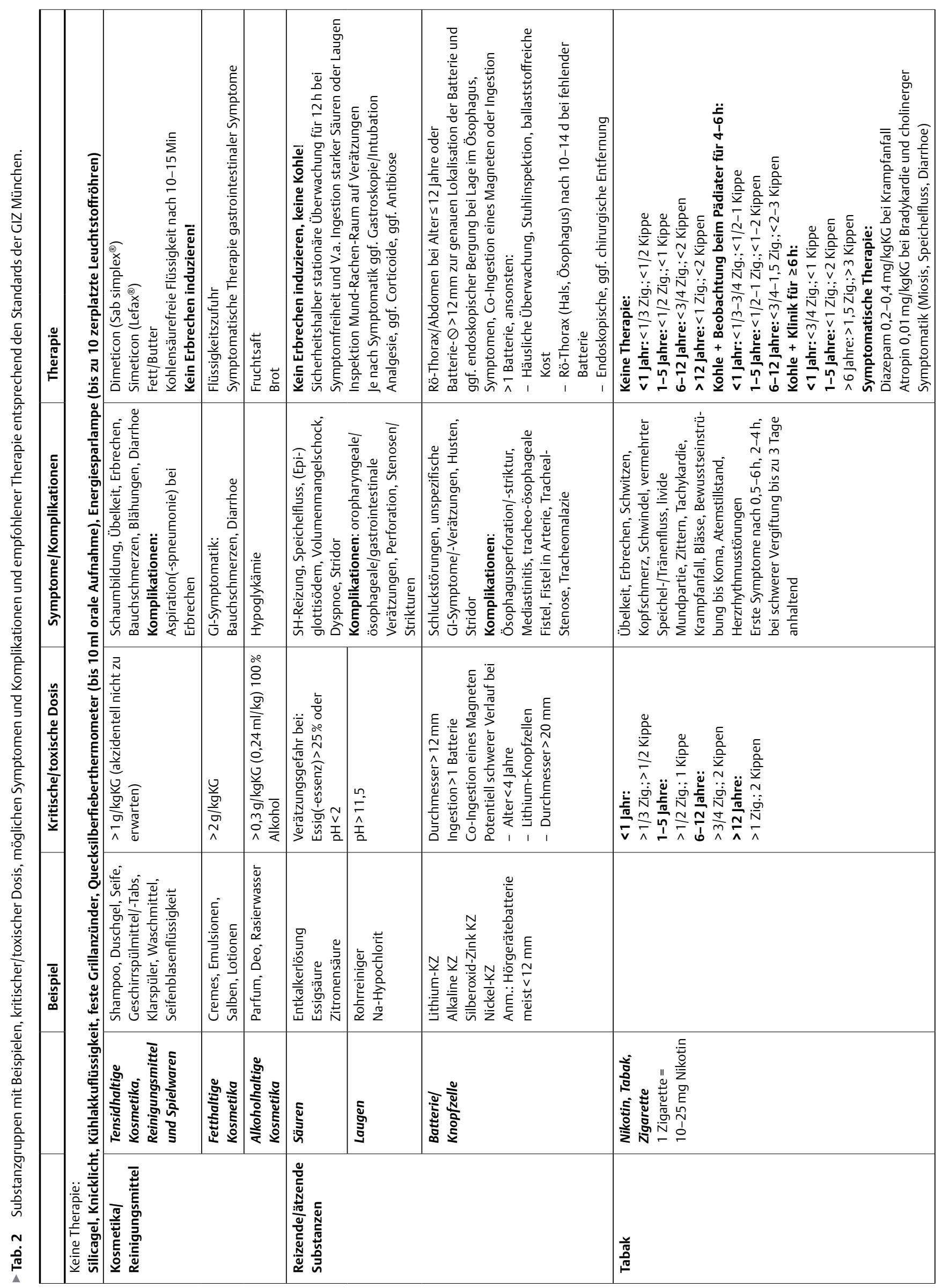




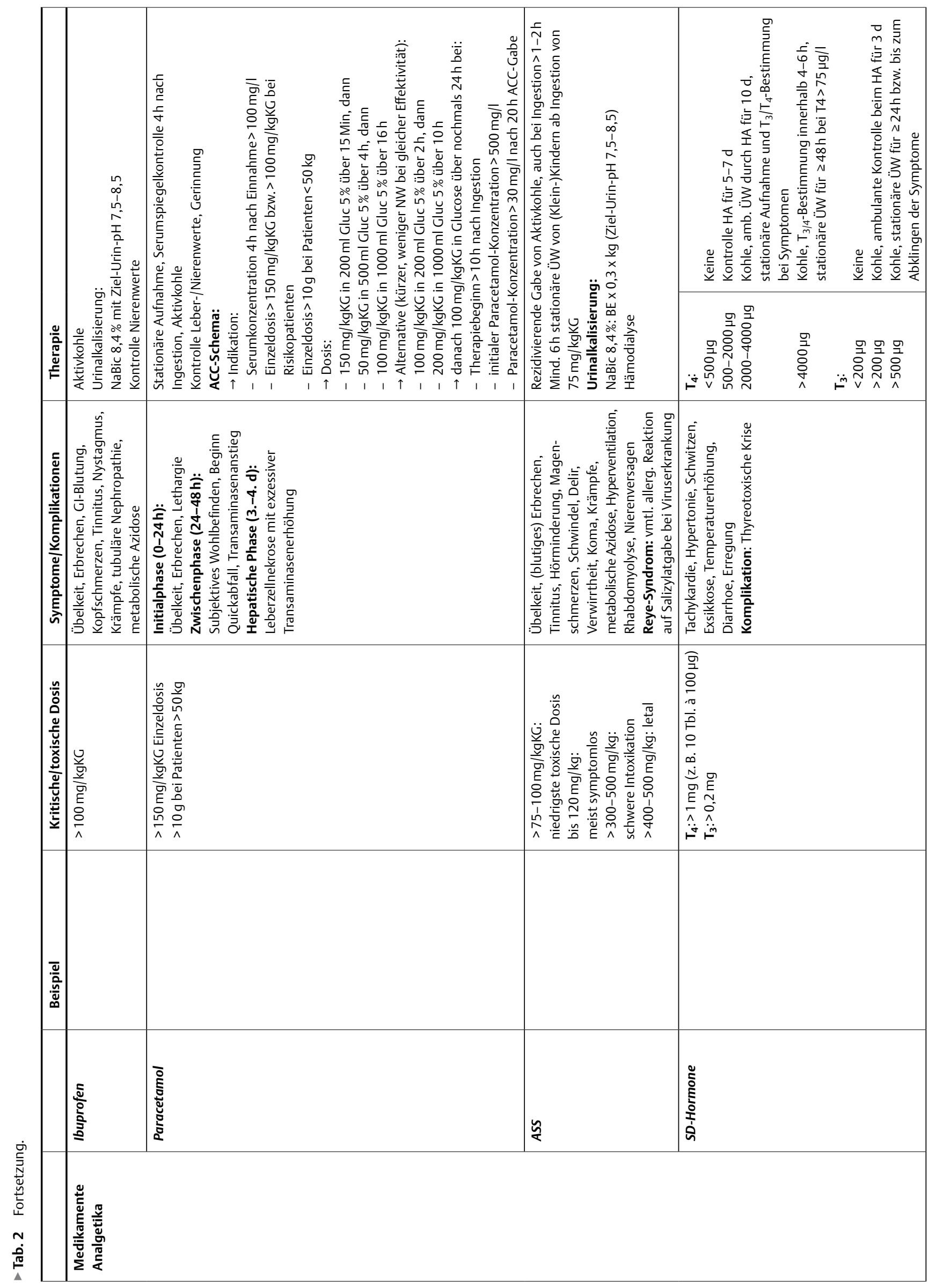




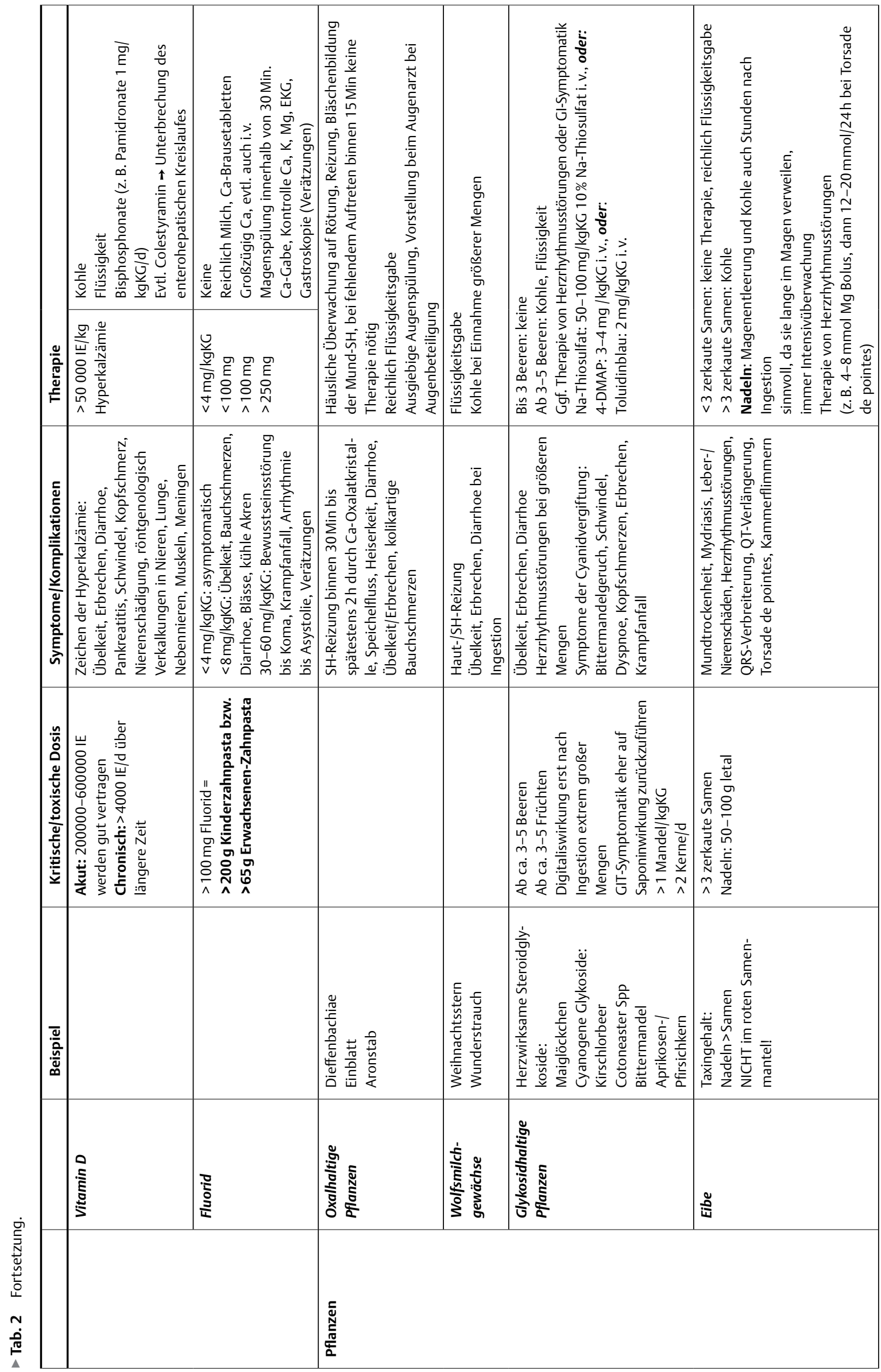


Verfügbarkeit des Internets, das oftmals wenig fundiertes Halbwissen liefert, gesteigertes Bedürfnis nach einer fachlich kompetenten, rund um die Uhr verfügbaren Anlaufstelle im Hinblick auf eine Beratung zu Risikoeinschätzung, Behandlung und Prävention von Vergiftungen sein. Aber auch eine generelle Sensibilisierung der Bevölkerung für medizinische Themen durch die Medien und nicht zuletzt der wachsende Bekanntheitsgrad der GIZen mit deren allseits verbreiteten Notfallnummern mögen hieran einen Anteil haben.

Die aus der bundesweiten Abfrage hervorgehenden Daten verdeutlichen sowohl angesichts der Dominanz der Altersgruppen $<7$ Jahre wie auch der großteils akzidentiellen Ätiologie der Vergiftungsfälle die Bedeutung präventiver Maßnahmen. Diese beinhalten insbesondere die Aufklärung von Eltern sowie mit der Beaufsichtigung und Erziehung von Kindern betrauter Personen. Informationen zur Prävention sollten potentielle Gefahrenquellen, aber auch Ersthelfermaßnahmen im akuten Vergiftungsfall berücksichtigen. Außerdem sollten sie auf die Möglichkeit einer präklinischen Verabreichung einfacher Antidota, wie Aktivkohle oder Entschäumer hinweisen und deren Bevorratung sowohl im häuslichen Umfeld als auch in Kindergärten, Schulen und öffentlichen Einrichtungen propagieren [7, 9, 11]. Aber auch Politik und Industrie sollten in diesem Punkt ihrer Verpflichtung nachkommen, Primärprophylaxe, z. B. durch verpflichtende Einführung kindersicherer Verschlüsse und Verpackungen (auch für Medikamente!), oder den Ersatz toxischer Substanzen durch weniger toxische Inhaltsstoffe in Reinigungs- und Haushaltsmitteln, auch umzusetzen [4].

In der Altersgruppe ab 14 Jahren spielen oftmals auch (para-) suizidal intendierte Intoxikationen mit Medikamenten eine zunehmende Rolle. Hier sind v. a. Analgetika, im jugendlichen Alter auch Psychopharmaka zu nennen und legen nahe, dass sowohl Ärzte wie Apotheker einen verantwortungsvollen Umgang beim Verschreiben bzw. der Abgabe von Medikamenten wahren sollten. Hier sollte auch eine konstruktive Zusammenarbeit mit der pharmazeutischen Industrie erfolgen - insbesondere in Bezug auf die Herstellung kleinerer Packungsgrößen.

Was den v. a. die Gruppe der Jugendlichen betreffenden Konsum legaler (Alkohol und Tabak) wie auch illegaler Drogen angeht, ist es neben der elterlichen Aufsichts- und Fürsorgepflicht schließlich auch Aufgabe der Politik, die Einhaltung der Abgabevorschriften, aber auch den Konsum zu überwachen und Regelverstöße zu sanktionieren.

\section{Schlussfolgerung}

Unsere GIZ-übergreifende Umfrage untermauert angesichts einer jährlich steigenden Anzahl an Anrufern die große Bedeutung der GIZen bei Ratsuchenden in Vergiftungsfragen. Bei der hier untersuchten Altersgruppe unter 18 Jahren nehmen Anrufe durch Laien die größte Gruppe der Anfragenden ein. Wir haben die häufigsten Vergiftungen bei Kindern und Jugendlichen und deren Ätiologie identifiziert, die sich bei Säuglingen, Klein-/Vorschul- und Schulkindern in der Regel akzidentiell, bei Jugendlichen hingegen häufiger missbräuchlich oder (para-)suizidal ereignen. Obwohl der Verlauf kindlicher Vergiftungen ganz überwiegend blande ist, darf gerade bei Intoxikationen in dieser Altersgruppe der wichtigste präventive Ansatz - nämlich die Vermeidung einer Vergiftung - durch vergleichsweise einfache und kostengünstige Maßnahmen nicht in den Hintergrund treten.

Unabhängig hiervon empfiehlt sich in jedem Vergiftungsfall auch auf Verdacht eine Rücksprache mit einer GIZ, um sich bzgl. der Einschätzung der Gefährdungssituation, der Indikation einer stationären Überwachung und ggf. der Indikation spezieller Therapiemaßnahmen beraten zu lassen.

\section{Limitationen}

Die Erfassung der Daten erfolgte anhand von Notrufprotokollen in den Datenbanken der beteiligten GIZen. Nachdem diese deutschlandweit nicht standardisiert sind, war eine einheitliche Datenerhebung in den ausgewerteten Kategorien nicht möglich. Während Geschlecht, Akuität, Ätiologie, Ingestionsweg und Schweregrad in allen GIZen für die jeweiligen Altersgruppen identisch erfasst werden, werden einzelne Noxen unterschiedlich dokumentiert, so dass hier die Bildung übergeordneter Kategorien bzw. die Identifikation der altersgruppenspezifisch häufigsten Noxen erforderlich war, denen die dokumentierten Fälle zahlenmäßig zugeordnet wurden.

\section{Stellungnahme zur Autorenschaft}

SG: design and initiation of the project, collection of data from the sites, analysis of data, manuscript writing SS: support with design and initation of the project, support with the collection of data from the sites, analysis of data, manuscript review MG: provision of data from the site, analysis of data, manuscript review AD, DM, SO, SA, TE, WR: provision of data from their site, manuscript review $\mathrm{FE}$ : supervision design and initiation process of the project, manuscript design and review

\section{Interessenkonflikt}

Die Autoren erklären hiermit, dass kein Interessenkonflikt besteht.

\section{Literatur}

[1] Benson BE, Hoppu K, Troutman WG et al. Position paper update: gastric lavage for gastrointestinal decontamination. Clinical toxicology (Philadelphia, Pa) 2013; 51: 140-146 Epub 2013/02/20. doi: 10.3109/15563650.2013.770154. PubMed PMID: 23418938

[2] Berlin G. Jahresbericht 2000. Beratungsstelle für Vergiftungserscheinungen und Embryonaltoxikologie, Berliner Betrieb für zentrale und gesundheitliche Aufgaben 2000

[3] Buckley NA, Eddleston M. The revised position papers on gastric decontamination. Clinical toxicology (Philadelphia, Pa) 2005; 43: 129-130 Epub 2005/04/13. PubMed PMID: 15822769; PubMed Central PMCID: PMCPMC1351142

[4] Eyer F, Zilker T. Expositionen mit Ätzstoffen. Ther Umsch. 2009; 66: 379-386

[5] Felgenhauer N, Zilker T. [Emergencies in general practice. Acute poisoning with medicinal drugs]. MMW Fortschritte der Medizin 2002; 144: 42-45 Epub 2002/07/16. PubMed PMID: 12116553

[6] Giftinformationszentrum der Länder Rheinland-Pfalz und Hessen KT. Jahresbericht 2011. GIZ Universität Mainz. 2011 
[7] Lamminpaa A, Vilska J, Hoppu K. Medical charcoal for a child's poisoning at home: availability and success of administration in Finland. Human \& experimental toxicology 1993; 12: 29-32 Epub 1993/01/01. doi: 10.1177/096032719301200106. PubMed PMID: 8094967

[8] München G. Jahresbericht 2016. Toxikologische Abteilung der II Medizinischen Klinik, Klinikum rechts der Isar. 2016

[9] Pfab R, Schmoll S, Dostal G et al. Single dose activated charcoal for gut decontamination: Application by medical non-professionals a prospective study on availability and practicability. Toxicology reports 2017; 4: 49-54 Epub 2017/09/30. doi: 10.1016/j.toxrep. 2016.12.007. PubMed PMID: 28959624; PubMed Central PMCID: PMCPMC5615092
[10] Schrettl V, Eyer F. Prävention in der klinischen Toxikologie. Bayerisches Ärzteblatt 2013; 12/2013: 632-638

[11] Spiller HA, Rodgers GC Jr. Evaluation of administration of activated charcoal in the home. Pediatrics 2001; 108: E100 Epub 2001/12/04. PubMed PMID: 11731627

[12] ToxInfoSuisse. Jahresberichte [cited 2017 15.12.2017]. Available from: http://toxinfo.ch/jahresberichte-neu_de

[13] Vale JA, Kulig K. Position paper: gastric lavage. Journal of toxicology Clinical toxicology 2004; 42: 933-943 Epub 2005/01/12. PubMed PMID: 15641639

\section{Hinweis}

Dieser Artikel wurde gemäß des Erratums vom 08.04.2022 geändert.

\section{Erratum}

Im obengenannten Artikel befand sich ein Fehler in $\$ Tab. 1 . 